\title{
Percutaneous Spinal Cord Stimulation Lead Placement Under Deep Sedation and General Anesthesia
}

\author{
Jamal Hasoon (D) - Ivan Urits · Omar Viswanath · Giustino Varrassi • \\ Thomas T. Simopoulos • Lynn Kohan · Genaro Gutierrez • \\ Vwaire Orhurhu $\cdot$ Musa Aner · Jatinder Gill
}

Received: July 20, 2021 / Accepted: September 28, 2021 / Published online: October 15, 2021

(C) The Author(s) 2021

\begin{abstract}
Introduction: Spinal cord stimulation (SCS) is a commonly utilized therapy for the treatment of neuropathic pain conditions. The Neurostimulation Appropriateness Consensus Committee (NACC) has recommended that the placement of percutaneous SCS leads be performed in an awake patient capable of providing feedback. It is not currently known how commonly this recommendation is adhered to by physicians in clinical practice. This article presents the findings of a survey designed to answer this important question.
\end{abstract}

J. Hasoon ( $ه)$ I I. Urits · T. T. Simopoulos · J. Gill Department of Anesthesiology, Critical Care, and Pain Medicine, Beth Israel Deaconess Medical Center, Harvard Medical School, 330 Brookline Avenue, Boston, MA 02115, USA

e-mail: Jhasoon@psadocs.com

J. Hasoon · G. Gutierrez

Pain Specialists of America, Austin, TX, USA

I. Urits · O. Viswanath

Department of Anesthesiology, Louisiana State

University Health Shreveport, Shreveport, LA, USA

O. Viswanath

Valley Anesthesiology and Pain Consultants,

Envision Physician Services, Phoenix, AZ, USA

O. Viswanath

Department of Anesthesiology, University of

Arizona College of Medicine Phoenix, Phoenix, AZ, USA
Methods: We conducted a survey of the active membership of the American Society of Regional Anesthesia and Pain Medicine (ASRA) and the Spine Intervention Society (SIS) regarding practice patterns with SCS therapy. We analyzed the percent of respondents who indicated that they use deep sedation and general anesthesia during SCS placement as well as any reported complications.

Results: Many practitioners frequently utilize deep sedation as well as general anesthesia when performing SCS implants. Our findings demonstrate that $77 \%$ of physicians reported that they utilize deep sedation for permanent SCS implants at times, and $45 \%$ of physicians

O. Viswanath

Department of Anesthesiology, Creighton

University School of Medicine, Omaha, NE, USA

G. Varrassi

PaoloProcacci Foundation, 00193 Roma, Italy

L. Kohan

Department of Anesthesia, Critical Care, and Pain Medicine, University of Virginia Medical Center, Charlottesville, VA, USA

V. Orhurhu

Department of Anesthesiology and Perioperative Medicine, University of Pittsburgh Medical Center, Williamsport, PA, USA

\section{Aner}

Department of Anesthesia, Critical Care, and Pain Medicine, Dartmouth-Hitchcock Medical Center, Dartmouth Medical School, Lebanon, NH, USA 
reported the use of general anesthesia for $10 \mathrm{kHz}$ implants. Additionally, 94\% of physicians reported that they have never had a complication related to the use of general anesthesia for a spinal cord stimulator placement.

Conclusions: This survey provides initial data on SCS practices among a large cohort of clinicians who utilize SCS. SCS lead placement under deep sedation and general anesthesia appears to be common practice for many physicians who perform implants. This survey should stimulate further research on this topic, given that the current safety guidelines and the rate of physicians reporting the use of deep sedation and general anesthesia for spinal cord stimulator placement remain at odds.

Keywords: Spinal cord simulation; Neuromodulation; Cylindrical electrodes; $10 \mathrm{kHz}$ stimulation; Chronic pain; Patient safety

\section{Key Summary Points}

The Neurostimulation Appropriateness Consensus Committee (NACC) safety guidelines for the reduction of severe neurological injury recommend that the placement of percutaneous spinal cord stimulation (SCS) leads be performed in an awake and conversant patient.

SCS implants have traditionally been performed with paresthesia mapping, which requires an awake and cooperative patient who will serve as a good monitor and alert the clinician in the case of needle or lead advancement into a nerve or the spinal cord.

The advent of anatomic lead placement without the need for paresthesia overlap and the increasing availability of intraoperative neuromonitoring allows for the option of elective deep sedation and general anesthesia for placement of percutaneous SCS leads.
There are risks and benefits when placing SCS leads in both awake patients and patients under deep sedation and general anesthesia.

This survey provides initial data on the practice parameters of physicians who utilize SCS therapy including the use of deep sedation and general anesthesia when performing SCS implants, as well as complications associated with the use of anesthesia.

\section{INTRODUCTION}

Spinal cord stimulation (SCS) is an increasingly utilized therapy that is effective for the treatment of refractory chronic neuropathic pain conditions [1]. The primary indication for SCS therapy is for the treatment of post-laminectomy syndrome as well as complex regional pain syndrome (CRPS) [2]. However, recent advances have broadened the scope of SCS therapy for a variety of other neuropathic pain conditions [3-9]. SCS provides pain relief by modulation of the pain pathway using electrical current through electrode leads. SCS is considered to be a safe therapy for chronic pain conditions with a low incidence of serious adverse events $[3,10]$.

SCS implants have traditionally been performed with paresthesia mapping, which requires an awake and cooperative patient. The rate of neurological injury after SCS placement has been shown to be very low, but a recent database analysis has placed this rate as high as 2.3\% [11]. The Neurostimulation Appropriateness Consensus Committee (NACC) safety guidelines for the reduction of severe neurological injury have recommended that the placement of percutaneous SCS leads be performed in an awake and conversant patient [12]. These guidelines assume that a responsive patient will serve as a good monitor and alert the clinician in case of needle or lead advancement into a nerve or the spinal cord. In 
addition, it would also alert a clinician if lead placement creates nerve or spinal cord compression. However, the guidelines do acknowledge that there may be circumstances in which the benefit of deep sedation or general anesthesia during percutaneous lead placement outweighs the risks. These circumstances may include a patient with a difficult airway likely to obstruct, high-dose opioid usage with difficulty in sedation, severe anxiety, movement disorders, or patient agitation with varying levels of sedation [13-17]. The guidelines state that when the risk-to-benefit ratio favors deep sedation or general anesthesia, then intraoperative neurophysiological monitoring (IONM) recommendations for paddle lead placement under deep sedation or general anesthesia should be considered [12]. Since a cooperative patient is required to assess the paresthesia generation and dermatomal overlap, the guidelines and procedural requirements are in alignment and easy to adhere to. However, with the advent of anatomic placement without the need for paresthesia overlap, as in $10 \mathrm{kHz}$ stimulation, and the increasing availability of IONM, the option of elective deep sedation and general anesthesia for placement of percutaneous SCS leads is increasingly viable. In these circumstances, the goals of the procedure and NACC recommendations no longer align. Although placing SCS leads in a responsive patient would be the best practice, this is often not feasible or predictable when performing this procedure under monitored anesthesia care (MAC) in the prone position.

We designed a survey to evaluate the practice parameters of physicians who utilize SCS therapy, including questions regarding the use of deep sedation and general anesthesia when performing SCS implants as well as complications associated with the use of anesthesia. We chose this subset of the survey as a separate article, as we felt it critical to report the percentage of physicians who are utilizing deep sedation and general anesthesia for SCS lead placement, which contrasts the current NACC recommendations.

\section{METHODS}

A survey related to various aspects of SCS practice was submitted and approved by the Institutional Review Board at Beth Israel Deaconess Medical Center. The survey was designed by the authors based upon perceived clinical importance and interest in the SCS community. The survey was then approved by the boards of the American Society of Regional Anesthesia and Pain Medicine (ASRA) and the Spine Intervention Society (SIS). This survey was sent as an email with a SurveyMonkey link to practitioners with active membership in these societies. The prospective recipients were invited for the survey by an email requesting their anonymous participation in a survey by clicking on a link. The recipients were informed that the survey concerned the practice parameters of pain physicians who perform spinal cord stimulator trials or implants. The recipients were asked to not complete the survey if they had already done so, since a significant number of recipients may have been member of both societies. The survey could not be sent to a dedicated neuromodulation society because of logistical issues.

Given the disparate aspects of SCS therapy that the queries pertained to, important clinical aspects were grouped together and will be submitted for publication separately. Here we present our results for technical aspects of SCS lead insertion. The three questions related to this were as follows:

(1) For permanent implants, do you use deep sedation (nonresponsive) $N=175$

(2) For $10 \mathrm{kHz}$ spinal cord stimulator implantation do you ever use general anesthesia? $N=165$

(3) Have you ever had a spinal cord stimulator complication related to the use of general anesthesia (such as nerve injury)? $N=186$

\section{RESULTS}

The results for the three questions are presented below. Survey responses were received between March 20, 2020, and June 26, 2020. The survey was delivered to 2967 members of SIS, with 
1259 opening the email, and 3169 members of ASRA, with 1477 opening the email. A total of 175 responded to question 1,165 responded to question 2 , and 186 responded to question 3 . The proportion and confidence interval for those who responded to the questions above is presented in Table 1.

Results from question 1 demonstrate that $32.6 \%$ of physicians often utilize deep sedation for permanent SCS placement, while $15.4 \%$ of physicians always utilize deep sedation for permanent SCS placement. Overall, 77\% of physicians report that they utilize deep sedation for permanent SCS implants. These results demonstrate that a majority of responding physicians are utilizing deep sedation for SCS placement at times. (Fig. 1).

Results from question 2 demonstrate that $45 \%$ of physicians report the use of general anesthesia for $10 \mathrm{kHz}$ implants. Ten-kilohertz SCS is paresthesia-free and is routinely placed anatomically without the need for paresthesia testing for placement. These results suggest that nearly half of implanting physicians utilize general anesthesia for $10 \mathrm{kHz}$ implantation (Fig. 2).

Regarding complications, roughly $6 \%$ of physicians reported a complication related to the use of general anesthesia for SCS placement (Table 1). A review of individualized responses for complications related to the use of general anesthesia revealed the following information, summarized in Table 2.

\section{DISCUSSION}

SCS has become a more commonly utilized therapy for intractable neuropathic pain conditions. Current safety guidelines recommend that implantation of percutaneous leads be performed in an awake and cooperative patient for confirmation of adequate placement as well as avoiding neurological injury [12]. Though there may be enhanced safety when the patient is awake, there are disadvantages to awake placement of SCS leads, including patient discomfort, variable levels of sedation used by providers, patient agitation and movement during the procedure, reliability of patient response, increased stress to the surgical team, and increased operative time [13-17]. Upon review of the survey results, it is apparent that physicians are commonly performing SCS lead placement under deep sedation and general anesthesia. Our results demonstrate that up to $77 \%$ of reporting physicians utilize deep sedation when implanting SCS devices and up to $45 \%$ use general anesthesia when implanting anatomically placed $10 \mathrm{kHz}$ systems, and this likely represents the limitations of performing the procedure in awake patient under light sedation.

Below we will discuss the known incidence of neurological complication related to SCS placement and discuss the advantages and disadvantages of performing SCS implants under deep sedation or general anesthesia. We also provide a rationale for the choices that physicians may make for determining an anesthetic and operative plan in these patients.

\section{Incidence and Causes of Neurological Complications Related to SCS Lead Insertion}

The true incidence of neurological complications related to SCS is unknown. Two large studies by Cameron and Mekhail et al. did not report any spinal cord injuries related to SCS placement $[18,19]$, but one must keep in mind that injuries may not be reported, and hence the true incidence is unknown. Petraglia et al. recently reported an overall risk of spinal cord injury within 30 days after percutaneous lead insertion of $2.35 \%$. However, this study was conducted by reviewing spinal cord injury codes used in the 30-day surgical period and could have overestimated the overall risk, as the codes used were not confirmed for accuracy [11]. In contrast to the low reported incidence of spinal cord injury in other studies in relation to SCS procedure, the American Society of Anesthesiologists (ASA) closed claims analysis of injuries relating to cervical procedures from 2005 to 2008 reported 20 cases of direct needle trauma to the spinal cord, and direct trauma accounted for $31 \%$ of all cases [20]. 
Table 1 Survey results along with the proportion and confidence intervals for those who responded

\begin{tabular}{|c|c|c|c|c|c|c|c|c|}
\hline & \multicolumn{2}{|c|}{ Never (95\% CI) } & \multicolumn{2}{|c|}{ Sometimes $(95 \% \mathrm{CI})$} & \multicolumn{2}{|c|}{ Often $(95 \% \mathrm{CI})$} & \multicolumn{2}{|c|}{ Always (95\% CI) } \\
\hline \multirow[t]{2}{*}{ Deep sedation, $N=175$} & \multicolumn{2}{|c|}{$23.4(17.3-30.4)$} & \multicolumn{2}{|c|}{$28.6(22-35.9)$} & \multicolumn{2}{|c|}{$32.6(25.7-40.1)$} & \multicolumn{2}{|c|}{$15.4(10.4-21.7)$} \\
\hline & \multicolumn{2}{|l|}{41} & \multicolumn{2}{|l|}{50} & \multicolumn{2}{|l|}{57} & \multicolumn{2}{|l|}{27} \\
\hline \multirow[t]{3}{*}{$10 \mathrm{kHz}, N=165$} & \multicolumn{2}{|c|}{$55.2(47.2-62.9)$} & \multicolumn{2}{|c|}{$21.2(15.2-28.3)$} & \multicolumn{2}{|c|}{$14.6(9.6-20.9)$} & \multicolumn{2}{|c|}{$9.1(5.2-14.6)$} \\
\hline & \multicolumn{2}{|l|}{91} & \multicolumn{2}{|l|}{35} & 24 & & \multicolumn{2}{|l|}{15} \\
\hline & & $0(95 \%$ & & $1(95 \% \mathrm{CI})$ & $2(95 \% \mathrm{CI})$ & $3(9$ & $\% \mathbf{C I})$ & $>5(95 \% \mathrm{CI})$ \\
\hline \multirow{2}{*}{\multicolumn{2}{|c|}{ Complication nerve injury, $N=186$}} & \multicolumn{2}{|c|}{$94.1(89.7-97)$} & $3.8(1.5-7.6)$ & $1.1(0.1-3.8)$ & \multicolumn{2}{|c|}{$0.5(0.01-0.3)$} & $0.5(0.01-0.3)$ \\
\hline & & 175 & & 7 & 2 & 1 & & 1 \\
\hline
\end{tabular}

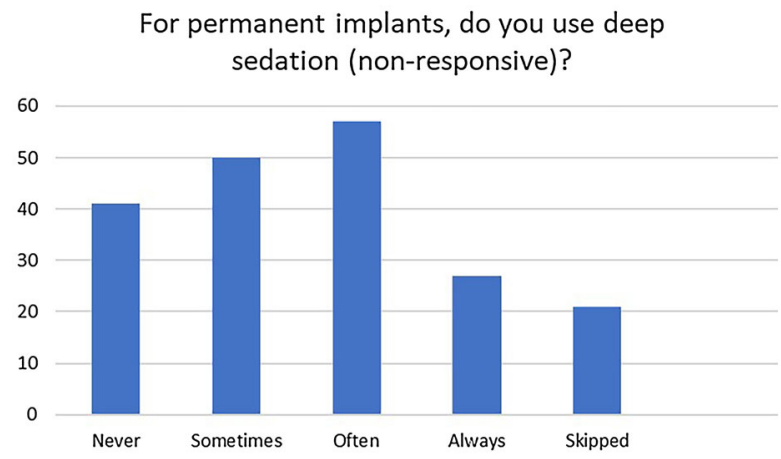

Fig. 1 Number of responding physicians who utilize deep sedation for permanent SCS implants

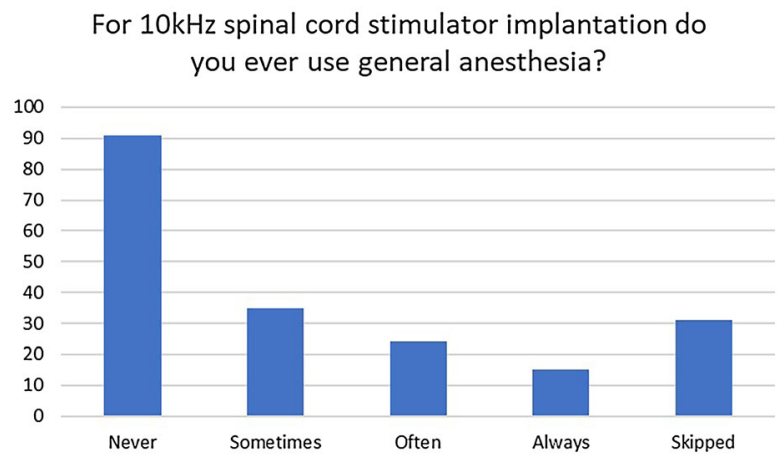

Fig. 2 Number of responding physicians who utilize general anesthesia for permanent $10 \mathrm{kHz}$ SCS implants

The causes of neurological injury during the SCS procedure may relate to direct injury to the spinal cord during needle insertion, compression of the spinal cord due to mass effect, or epidural hematoma or abscess. Among these complications, the most devastating is inadvertent needle/lead placement into the spinal cord during epidural localization. Thus it is essential to review the target anatomy, proceed cautiously with epidural placement, and optimize visualization with fluoroscopy (including contralateral views if necessary) to decrease these risks. This may happen due to poor needle control, ineffective use of fluoroscopy, or other factors such as patient movement. The best protection against direct neurological injury during spinal cord stimulator lead insertion is a review of the target anatomy, great degree of care in epidural localization, and appropriate use of fluoroscopy to clearly visualize the dorsal epidural space such as the contralateral oblique view, especially with cervical lead placement [21]. If these safeguards fail, an awake patient may be the last line of defense against neurological injury. In the ASA claims, $67 \%$ of patients with a neurological injury were deeply sedated, demonstrating that deep levels of sedation may be a risk factor, as a sedated patient cannot alert the provider of possible neurological compromise [20]. On the other hand, performing SCS in a lightly sedated and anxious patient who is prone to sudden movements may also carry very high risk, and careful analysis of the risks and benefits may show that deep sedation and an immobile patient would be a safer scenario in some cases.

IONM is a valuable option to replace the paresthesia mapping that is done in an awake 
Table 2 Review of individualized responses for complications related to the use of general anesthesia (GA)

\begin{tabular}{lcll}
\hline & $\begin{array}{l}\text { No. of SCS complications } \\
\text { related to the use of GA }\end{array}$ & $\begin{array}{l}\text { SCS trials performed } \\
\text { per year }\end{array}$ & $\begin{array}{l}\text { SCS implants } \\
\text { performed per year }\end{array}$ \\
\hline Physician 1 & 1 & $11-20$ & $5-10$ \\
Physician 2 & 1 & $11-20$ & $<5$ \\
Physician 3 & 1 & $11-20$ & $11-20$ \\
Physician 4 & 1 & $5-10$ & $5-10$ \\
Physician 5 & 1 & $21-50$ & $21-50$ \\
Physician 6 & 1 & $11-20$ & $11-20$ \\
Physician 7 & 1 & $11-20$ & $11-20$ \\
Physician 8 & 2 & $>50$ & $>50$ \\
Physician 9 & 2 & $5-10$ & Question skipped \\
Physician 10 & 3 & $11-20$ & $11-20$ \\
Physician 11 & 5 & $>50$ & $>50$ \\
\hline
\end{tabular}

patient. With the use of IONM, physicians can evaluate myotomal coverage and use this as a surrogate for dermatomal coverage [22]. However, given that the lead is already placed, this technique would not protect against inadvertent needle injury or erroneous lead placement. Thus, the purpose of IONM is primarily to allow paresthesia mapping but cannot serve as a tool to detect cord injury at the time of placement.

To summarize, although SCS is a very safe procedure, the true incidence of serious procedure-related neurological complications and contributing factors is unknown. While there is some evidence that deep sedation may increase the risk of complications, it is extrapolated from other databases. Given that light sedation may often not be adequate to perform a SCS procedure, our study demonstrates that $77 \%$ of physicians use deep sedation for SCS and 45\% use general anesthesia for $10 \mathrm{kHz}$ SCS placement, and of these, $6 \%$ had experienced a spinal cord stimulator complication related to the use of general anesthesia. Individualized responses have been provided in Table 2 . However, we did not collect details on specific complications, and it is unclear whether these are anesthetic complications related to providing general anesthesia in the prone position, or procedural complications such as neurological injury. Additionally, while $6 \%$ of the responders noted a complication, the actual overall risk of injury is unknown, as we do not know the total SCS case numbers for all physician responders. Similar to the studies by Cameron and Mekhail et al. $[18,19]$, the risk of neurological injury related to SCS placement is likely very low. Given the small sample size, the significance of this number is unclear.

With this background we will analyze the pros and cons of various approaches.

\section{Light Sedation in a Cooperative Patient}

Traditionally, percutaneous SCS leads are inserted under light sedation, and paresthesia mapping is performed unless anatomic placement is planned. The leads are then tunneled and the implantable pulse generator (IPG) implanted. This can be performed via two commonly used methods. One technique utilizes an incision first under generous local anesthesia up to the desired depth, followed by lead insertion into the epidural space. 
Alternatively, the physician can first place the leads in the desired location followed by an incision, and then the leads are withdrawn into the incision.

The advantage of light sedation is that the leads can be placed and the patient may serve as their own monitor, paresthesia mapping can be performed, and the sedation may be deepened after the leads have been implanted. However, this approach has a very real disadvantage in that it is often not feasible. Patients must often be deeply sedated prior to lead insertion because of several factors such as anxiety, inability to remain still, pain despite adequate local anesthesia, discomfort of lying in the prone position, and claustrophobia, to name a few. This is especially relevant in cervical lead placement when immobility and true midline neck alignment are important. This approach is always tentative, and levels of sedation may fluctuate during the procedure. Additionally, light sedation may be used for lead placement, but deepening afterwards may at times create an unpredictable and unsafe anesthetic. ASA closed claims data show significant risk of respiratory depression and unpredictability in depth of anesthetic with MAC cases. Risk of death and brain damage in MAC cases was similar to that in cases with general anesthesia [23]. Risk of complications with prone MAC cases may be even higher because of airway inaccessibility (Table 3).

Thus, the very real advantage of light sedation is often lost in practical application. Consistent with these observations, only $23 \%$ of the recipients always avoided deep sedation with an unresponsive patient for permanent implantation. In terms of what one can do in one's own practice, avoiding deep sedation is a good practice to minimize the risk of neurological complications and is consistent with NACC guidelines. However, this is only adhered to by a quarter of this cohort. As previously discussed, in many clinical scenarios the NACC guidelines may not be feasible and the use of deep sedation may be required, which has been demonstrated and is consistent with the majority of surveyed physicians.

\section{Deep Sedation with an Unresponsive Patient}

Deep sedation for SCS lead insertion essentially provides general anesthesia conditions in a patient with an unprotected airway. The main reason this is commonly used is that it is very often needed to accomplish the goals of the procedure while still being able to test for paresthesia coverage. Even though it allows for paresthesia mapping, the testing in this state is often time-consuming and less than optimal. One must keep in mind that deep sedation with an uncontrolled airway in prone position is often not the safest option, and as noted before, MAC has been associated with significant and serious complications including death and brain damage [23]. The finding that the majority of responders utilize deep sedation at times despite the disadvantages points to the fact that this is a realistic alternative when paresthesia mapping may be done with the patient serving as a guide. In terms of one's own practice, having a patient undergo deep sedation is not

Table 3 Summary of the advantages and disadvantages of utilizing light sedation

\begin{tabular}{ll}
\hline Advantages & Disadvantages \\
\hline Allows for good paresthesia testing & Fluctuating levels of sedation \\
Patient can serve as a monitor & Patient discomfort \\
Less possibility of respiratory compromise & May required deeper levels of sedation due to patient discomfort \\
Avoids general anesthesia & Inadvertent patient movement \\
& Disinhibition \\
\hline
\end{tabular}


Table 4 Summary of the advantages and disadvantages of utilizing deep sedation

\begin{tabular}{ll}
\hline Advantages & Disadvantages \\
\hline Allows for surgery to be done while keeping the possibility of paresthesia & May be a challenging anesthetic \\
mapping in a patient who can tolerate lighter levels of sedation & Fluctuating levels of sedation \\
Avoids general anesthesia & Uncontrolled airway \\
Patient may still be able to alert of impending neurotrauma & Respiratory Depression \\
& May need to pack the wound and flip the \\
patient intraoperative & Unreliable paresthesia testing \\
& Increased operative time-patient waking \\
for testing
\end{tabular}

consistent with the recommendations of NACC but is consistent with the practices of this cohort. One must carefully decide, case by case and in consort with the anesthesiologist, as to how to proceed with the case, weighing the feasibility of light sedation (Table 4).

\section{General Anesthesia}

We asked whether physicians performed general anesthesia for SCS lead placement only for $10 \mathrm{kHz}$ stimulation, since the placement is anatomic and does not require paresthesia mapping. The finding that $45 \%$ of responders chose to use utilize general anesthesia at times points to the fact that there are very real drawbacks to MAC and awake placement of SCS leads [23]. Studies have shown that utilizing anatomic placement of leads allows for a smoother procedural experience for the surgeon and a faster procedural time [24-26]. Potential advantages would include less operating room time, decreased staff requirements, and possibly a decreased rate of surgical site infections [27]. The drawbacks of general anesthesia are the inability to detect and avoid neurological injury when the needle or leads enter or compress neural tissue, as the patient is no longer able to serve as a warning. Having an alert and oriented patient allows for quick identification of potential neural compromise with interpretation of pain or paresthesias $[12,28]$. In terms of one's own practice, each physician must

Table 5 Summary of the advantages and disadvantages of utilizing general anesthesia

\begin{tabular}{ll}
\hline Advantages & Disadvantages \\
\hline Patient comfort & Patient cannot alert and serve as a monitor of neurological compromise \\
Faster & Traditional paresthesia testing not available \\
Still surgical field & \\
Controlled airway & \\
Safer anesthetic in patients difficult to sedate & \\
\hline
\end{tabular}


carefully consider the advantages and disadvantages of general anesthesia, and the decision may be made case by case. Although not recommended by NACC guidelines, $25 \%$ of physicians are often or always performing general anesthesia for $10 \mathrm{kHz}$ SCS lead placement, and NACC guidelines appear to differ from the community standard of care (Table 5).

Furthermore, the availability of IONM extends the possibility of general anesthesia for all SCS placements and negates the need for an alert and cooperative patient. This is especially true in view of the expanding indications for SCS [29] and/or the need to combine different techniques to obtain good pain control [30].

\section{Limitations}

This study presents the anesthetic choices of a large cohort of physicians practicing SCS therapy and is a first report of this type. There are several limitations that we would also like to address. This survey was submitted to members of SIS and ASRA through internal email registries to collect practice patterns for percutaneous cylindrical SCS leads. These are overwhelmingly non-surgical societies, and the data obtained would thus pertain to cylindrical percutaneous spinal cord stimulator leads. It is possible that surgical subspecialists implanting paddle leads with membership in these societies could have inadvertently participated in this survey; if so, this number would be exceedingly low. Additionally, these societies are not primarily neuromodulation societies, and have membership from varied disciplines. Not all members of these societies perform neuromodulation, and there may be overlapping membership between societies. Thus, the true responder rates of physicians who perform neuromodulation is ultimately not known. However, we did not adjust for any overlap in the membership, leading to a possible lower number of total recipients and higher response rate, since the magnitude of overlap was not known. Additionally, this may explain why our responder rate was low for this survey.

Since this is a descriptive survey that is looking for objective practice patterns of physicians who utilize neuromodulation, we believe that individuals more likely to respond to this survey are those who utilize this therapy in their practice and are unlikely to skew the results. Ultimately, we believe this survey provides valuable information to interventional physicians looking at the practice patterns of their peers. This survey also provides evidence that deep sedation and general anesthesia for permanent SCS placement is common practice amongst interventional pain physicians and their peers.

\section{CONCLUSION}

SCS has typically been performed with an awake patient utilizing paresthesia mapping. NACC recommends that lead placement be performed in a responsive patient. This survey demonstrates that SCS is commonly being performed under deep sedation and general anesthesia. Our findings demonstrate that $77 \%$ of physicians reported that they utilize deep sedation for permanent SCS implants, with $45 \%$ of physicians reporting the use of general anesthesia for $10 \mathrm{kHz}$ implants. This may reflect the fact that it is often not feasible to do these cases under light sedation, and general anesthesia offers some advantages. The true rate and causes of neurological complications and the contribution of deep sedation to this during SCS placement is unknown; similarly, the true rate of complications related to providing MAC in the prone position is also unknown. This survey should stimulate further research on this topic and potentially serve as a foundation to revisit the current safety guidelines, as a significant proportion of physicians who perform implantation appear to be utilizing deep sedation and general anesthesia for percutaneous SCS placement.

\section{ACKNOWLEDGEMENTS}

Funding. No funding and/or sponsorship was received in relation to this paper. 
Authorship. All named authors meet the International Committee of Medical Journal Editors (ICMJE) criteria for authorship for this article, take responsibility for the integrity of the work as a whole, and have given their approval for this version to be published.

Author Contributions. JH, IU, TS, LK, MA, JG: study design, data extraction, statistical analysis, manuscript drafting. OV, GV, GG, VO, MA: data extraction, manuscript revision.

Disclosures. Jamal Hasoon, Ivan Urits, Thomas $\mathrm{T}$ Simopoulos, Lynn Kohan, Genaro Gutierrez, Vwaire Orhurhu, Musa Aner, and Jatinder Gill have nothing to disclose. Giustino Varrassi is the journal's Editor and Omar Viswanath is a member of the journal's Editorial Board.

Compliance with Ethics Guidelines. The study was performed in accordance with the Helsinki Declaration of 1964 and subsequent amendments. Institutional review board (IRB) approval was obtained through Beth Israel Deaconess Medical Center. The prospective recipients were invited for the survey by an email requesting their anonymous participation in a survey by clicking on a link. The recipients were informed that the survey was regarding the practice parameters of pain physicians who perform spinal cord stimulator trials or implants.

Data Availability. The datasets analyzed during the current study are available from the corresponding author upon reasonable request.

Open Access. This article is licensed under a Creative Commons Attribution-NonCommercial 4.0 International License, which permits any non-commercial use, sharing, adaptation, distribution and reproduction in any medium or format, as long as you give appropriate credit to the original author(s) and the source, provide a link to the Creative Commons licence, and indicate if changes were made. The images or other third party material in this article are included in the article's Creative Commons licence, unless indicated otherwise in a credit line to the material. If material is not included in the article's Creative Commons licence and your intended use is not permitted by statutory regulation or exceeds the permitted use, you will need to obtain permission directly from the copyright holder. To view a copy of this licence, visit http://creativecommons.org/licenses/by$\mathrm{nc} / 4.0 /$.

\section{REFERENCES}

1. Kumar K, Taylor RS, Jacques L, et al. The effects of spinal cord stimulation in neuropathic pain are sustained; a 24-month follow-up of the prospective randomized controlled trial of the effectiveness of spinal cord stimulation. Neurosurgery. 2008;63: 762-70.

2. Taylor RS. Spinal cord stimulation in complex regional pain syndrome and refractory neuropathic back and leg pain/failed back surgery syndrome: results of a systematic review and meta-analysis. J Pain Symptom Manage. 2006;31(4SUPPL):13-9. https://doi.org/10.1016/j.jpainsymman.2005.12. 010.

3. Deer TR, Mekhail N, Provenzano D. The appropriate use of neurostimulation of the spinal cord and peripheral nervous system for the treatment of chronic pain and ischemic diseases: the Neuromodulation Appropriateness Consensus Committee. Neuromodulation. 2014;17:515-50.

4. Lee AW, Pilitsis JG. Spinal cord stimulation: indications and outcomes. Neurosurg Focus. 2006;21: E3.

5. Kapural L, Gupta M, Paicius R, Strodtbeck W, Vorenkamp KE, Gilmore C, Gliner B, Rotte A, Subbaroyan J, Province-Azalde R. Treatment of chronic abdominal pain with $10-\mathrm{kHz}$ spinal cord stimulation: safety and efficacy results from a 12-month prospective, multicenter, feasibility study. Clin Transl Gastroenterol. 2020;11(2):e00133.

6. Dawood Sayed MD, Jeffrey Foster DO, Ali Nairizi MD, Shawn Sills MD. $10 \mathrm{kHz}$ high-frequency spinal cord stimulation for chronic thoracic pain: a multicenter case series and a guide for optimal anatomic lead placement. Pain Physician. 2020;23: E369-76.

7. Berger AA, Hasoon J, Urits I, Viswanath O, Gill J. 10 $\mathrm{kHz}$ spinal cord stimulation for combined alleviation of post-laminectomy syndrome and chronic abdominal pain: a case report. J Pain Res. 2020;30(13):873-5. 
8. Al-Kaisy A, Van Buyten JP, Kapural L, Amirdelfan K, Gliner B, Caraway D, Subbaroyan J, Edgar D, Rotte A. $10 \mathrm{kHz}$ spinal cord stimulation for the treatment of non-surgical refractory back pain: subanalysis of pooled data from two prospective studies. Anaesthesia. 2020;75(6):775-84.

9. Al-Kaisy A, Palmisani S, Smith TE, Carganillo R, Houghton R, Pang D, Burgoyne W, Lam K, Lucas J. Long-term improvements in chronic axial low back pain patients without previous spinal surgery: a cohort analysis of $10-\mathrm{kHz}$ high-frequency spinal cord stimulation over 36 months. Pain Med. 2018;19(6):1219-26.

10. Verrills P, Sinclair C, Barnard A. A review of spinal cord stimulation systems for chronic pain. J Pain Res. 2016;9:481-92.

11. Petraglia FW, Farber SH, Gramer R, et al. The incidence of spinal cord injury in implantation of percutaneous and paddle electrodes for spinal cord stimulation. Neuromodulation. 2016;19:85-90.

12. Deer TR, Lamer TJ, Pope JE, Falowski SM, Provenzano DA, Slavin K, Golovac S, Arle J, Rosenow JM, Williams K, McRoberts P. The neurostimulation appropriateness consensus committee (NACC) safety guidelines for the reduction of severe neurological injury. Neuromodulation Technol Neural Interface. 2017;20(1):15-30.

13. Falowski SM, Celii A, Sestokas AK, Schwartz DM, Matsumoto C, Sharan A. Awake vs. asleep placement of spinal cord stimulators: a cohort analysis of complications associated with placement. Neuromodulation. 2011;14:130-4 (discussion 134-135).

14. Roth SG, Lange S, Haller J, et al. A prospective study of the intra- and postoperative efficacy of intraoperative neuromonitoring in spinal cord stimulation. Stereotact Funct Neurosurg. 2015;93:348-54.

15. Shils JL, Arle JE. Intraoperative neurophysiologic methods for spinal cord stimulator placement under general anesthesia. Neuromodulation. 2012;15:560-71 (Discussion 571-562).

16. Air EL, Toczyl GR, Mandybur GT. Electrophysiologic monitoring for placement of laminectomy leads for spinal cord stimulation under general anesthesia. Neuromodulation. 2015;15:573-80.

17. Mammis A, Mogilner AY. The use of intraoperative electrophysiology for the placement of spinal cord stimulator paddle leads under general anesthesia. Neurosurgery. 2012;70:230-6.

18. Cameron T. Safety and efficacy of spinal cord stimulation for the treatment of chronic pain: a 20-year literature review. J Neurosurg. 2004;100: 254-67.
19. Mekhail NA, Mathews M, Nageeb F, Guirguis M, Mekhail MN, Cheng J. Retrospective review of 707 cases of spinal cord stimulation: indications and complications. Pain Pract. 2011;11:148-53.

20. Rathmell JP, Michna E, Fitzgibbon DR, Stephens LS, Posner KL, Domino KB. Injury and liability associated with cervical procedures for chronic pain. Anesthesiology. 2011;114(4):918-26. https://doi. org/10.1097/ALN.0b013e31820fc7f2.

21. Gill JS, Aner M, Nagda JV, Keel JC, Simopoulos TT. Contralateral oblique view is superior to lateral view for interlaminar cervical and cervicothoracic epidural access. Pain Med. 2015;16(1):68-80.

22. Shils JL, Arle JE. Neuromonitoring for spinal cord stimulation lead placement under general anesthesia. J Clin Neurol. 2018;14(4):444-53. https:// doi.org/10.3988/jcn.2018.14.4.444.

23. Bhananker SM, Posner KL, Cheney FW, Caplan RA, Lee LA, Domino KB. Injury and liability associated with monitored anesthesia care: a closed claims analysis. Anesthesiology. 2006;104:228-34.

24. Al-Kaisy A, Baranidharan G, Palmisani S, Pang D, Will O, Wesley S, Crowther T, Ward K, Castino P, Raza A, Agnesi F. Comparison of paresthesia mapping to anatomical placement in burst spinal cord stimulation: initial trial results of the prospective, multicenter, randomized, double-blinded, crossover, CRISP study. Neuromodulation. 2020;23(5): 613-9.

25. Pope JE, Schu S, Sayed D, Raslan AM, Baranidharan G, Heros RD, Blomme B, Capobianco RA, Deer TR. Anatomic lead placement without paresthesia mapping provides effective and predictable therapy during the trial evaluation period: results from the prospective, multicenter, randomized, DELIVERY study. Neuromodulation. 2020;23(1):109-17.

26. Russo M, Van Buyten JP. 10-kHz high-frequency SCS therapy: a clinical summary. Pain Med. 2015;16(5):934-42.

27. Cheng H, Chen BP, Soleas IM, Ferko NC, Cameron CG, Hinoul P. Prolonged operative duration increases risk of surgical site infections: a systematic review. Surg Infect (Larchmt). 2017;18(6):722-35.

28. Neal JM, Barrington MJ, Brull R, et al. The second ASRA practice advisory on neurologic complications associated with regional anesthesia and pain medicine: executive summary 2015. Reg Anesth Pain Med. 2015;40:401-30.

29. Giglio M, Preziosa A, Rekatsina M, et al. Successful spinal cord stimulation for necrotizing Raynaud's phenomenon in COVID-19 affected patient: the 
nightmare comes back. Cureus. 2021. https://doi. org/10.7759/cureus. 14569 .

30. Berger A, Urits I, Hasson J, et al. Improved pain control with combination spinal cord stimulator therapy utilizing sub-perception and traditional paresthesia based waveforms: a pilot study. Anesth Pain Med. 2021;11(1): e113089. https://doi.org/10. 5812/aapm.113089. 